\title{
Metabolic costs induced by lactate in the toad Bufo marinus: new mechanism behind oxygen debt?
}

\author{
ILKA PINZ AND HANS-O. PÖRTNER \\ Alfred-Wegener-Institute for Polar and Marine Research, \\ 27568 Bremerhaven, Germany \\ Submitted 21 February 2002; accepted in final form 1 November 2002
}

Pinz, Ilka, and Hans-O. Pörtner. Metabolic costs induced by lactate in the toad Bufo marinus: new mechanism behind oxygen debt?. J Appl Physiol 94: 1177-1185, 2003. Firstpublished November 8,2002;10.1152/japplphysiol.00131. 2002.-The mechanism of an increase in metabolic rate induced by lactate was investigated in the toad Bufo marinus. Oxygen consumption $\left(\dot{\mathrm{V}}_{2}\right)$ was analyzed in fully aerobic animals under hypoxic conditions $\left(7 \% \mathrm{O}_{2}\right.$ in air), accompanied by measurements of catecholamines in the plasma, and was measured in isolated hepatocytes in vitro under normoxia by using specific inhibitors of lactate proton symport [ $\alpha$-cyano-4-hydroxycinnamate $(\mathrm{CHC})]$ and sodium proton exchange (EIPA). The rise in metabolic rate in vivo can be elicited by infusions of hyperosmotic (previous findings) or isosmotic sodium lactate solutions (this study). Despite previous findings of reduced metabolic stimulation under the effect of adrenergic blockers, the increase in $\mathrm{V}_{2}$ in vivo was not associated with elevated plasma catecholamine levels, suggesting local release and effect. In addition to the possible in vivo effect via catecholamines, lactate induced a rise in $\dot{\mathrm{V}}_{2}$ of isolated hepatocytes, depending on the concentration present in a weakly buffered Ringer solution at $\mathrm{pH} 7.0$. No increase was found at higher $\mathrm{pH}$ values $(7.4$ or 7.8 ) or in HEPES-buffered Ringer solution. Inhibition of the $\mathrm{Lac}^{-}-\mathrm{H}^{+}$ transporter with $\alpha-\mathrm{CHC}$ or of the $\mathrm{Na}^{+} / \mathrm{H}^{+}$exchanger with EIPA prevented the increase in metabolic rate. We conclude that increased $\dot{\mathrm{V}}_{2}$ at an elevated systemic lactate level may involve catecholamine action, but it is also caused by an increased energy demand of cellular acid-base regulation via stimulation of $\mathrm{Na}^{+} / \mathrm{H}^{+}$exchange and thereby $\mathrm{Na}^{+}-\mathrm{K}^{+}$ATPase. The effect depends on entry of lactic acid into the cells via lactate proton symport, which is likely favored by low cellular surface $\mathrm{pH}$. We suggest that these energetic costs should also be considered in other physiological phenomena, e.g., when lactate is present during excess, postexercise $\mathrm{V}_{2}$.

energy cost of acid-base regulation; excess postexercise oxygen consumption; hypoxia; lactate proton symport; oxygen consumption; sodium proton exchange

IT IS WELL KNOWN THAT METABOLIC RATE remains elevated after anaerobic exercise, indicating a partial repayment of an oxygen debt [also called excess postexercise oxygen consumption (EPOC)] $(6,11)$. More recently, it has been found that the metabolic rate can rise with the onset of hypoxia, accompanied by an accumulation

Address for reprint requests and other correspondence: H.-O. Pörtner, Alfred-Wegener-Institute for Polar and Marine Research, Columbusstrasse, 27568 Bremerhaven, Germany (E-mail: hpoertner @awi-bremerhaven.de). of lactate in the body fluids below a critical $\mathrm{PO}_{2}\left(\mathrm{P}_{\mathrm{c}}\right)(30$, 31 ). The rise in oxygen consumption below the $P_{c}$ was not only seen in an amphibian, Bufo marinus (31), but also in goldfish and rainbow trout (3). In all of these cases, the glycolytic end product lactate is present; however, the mechanisms causing the metabolic increment remain incompletely understood (e.g., Ref. 34).

Pörtner et al. (34) demonstrated a cause-and-effect relationship between lactate accumulation and the rise in oxygen consumption in $B$. marinus by systemic infusion of a hyperosmotic solution of sodium lactate. Oxygen consumption was increased as long as the levels of plasma lactate remained above $1.5-2 \mathrm{mmol} / \mathrm{l}$ at slightly elevated extracellular $\mathrm{pH}$, paralleled by unchanged or insignificantly reduced intracellular $\mathrm{pH}$ $\left(\mathrm{pH}_{\mathrm{i}}\right)$ in some tissues (Ref. 30 and H. O. Pörtner and S. C. Wood, unpublished observations). Only the effect of lactate could consistently explain the steady-state increase in metabolic rate $(34,36)$. The application of $\alpha$ - and $\beta$-adrenergic blockers before injection of sodium lactate prevented much (but not all) of the increase in oxygen consumption (34). At that time, it seemed likely that the lactate-induced increase in oxygen consumption was exclusively mediated through the effect of catecholamines. This interpretation is in line with the observation that several vertebrates, including toads, release catecholamines under severely hypoxic conditions $(1,23,27)$. When different experimental temperatures in the two available studies of the toad $B$. marinus are considered, it appears that onset of catecholamine (epinephrine and norepinephrine) accumulation in the plasma occurred at about the same environmental $\mathrm{Po}_{2}$ as found earlier for the accumulation of lactate $(1,34)$. In this context, it seemed likely that both the lactate anion and a decrease in $\mathrm{pH}$ could independently cause behavioral hypothermia, whereas the lactate anion only caused the increase in oxygen consumption $(8,9,12,34,41)$.

However, concomitant work by Watson et al. (44) indicated that lactate or lactic acid may also become effective at the cellular level. Exposure to lactic acidosis was associated with a stimulation of metabolic rate in isolated turtle cardiomyocytes. Watson et al. (44)

The costs of publication of this article were defrayed in part by the payment of page charges. The article must therefore be hereby marked "advertisement" in accordance with 18 U.S.C. Section 1734 solely to indicate this fact. 
found an increased oxygen consumption of the myocytes in acidic, extracellular solutions and suggested that this was caused by the concomitant drop in $\mathrm{pH}_{\mathrm{i}}$, which stimulated the $\mathrm{Na}^{+} / \mathrm{H}^{+}$exchanger and, thereby, $\mathrm{Na}^{+}-\mathrm{K}^{+}$-ATPase. The mechanism that caused the drop in $\mathrm{pH}_{\mathrm{i}}$ remained unidentified. Also, these findings do not help to explain the effect of increased oxygen consumption on lactate formation at maintained or even slightly elevated plasma $\mathrm{pH}$ values $(34,37)$. Further consideration revealed, however, that plasma $\mathrm{pH}$ is not necessarily a good indicator of $\mathrm{pH}$ at the cellular surface (37). Transmembrane proteins will rather be affected by interstitial or cellular surface $\mathrm{pH}$ values that differ from plasma $\mathrm{pH}$ and may also change differently according to the lower buffering capacity.

All of these questions led us to further investigate the mechanisms of lactate-induced metabolic stimulation in B. marinus. First of all, we complemented the previous work with adrenergic blockers (34) and analyzed whether increased plasma lactate levels cause a release of catecholamines, which in turn might cause a rise in oxygen consumption not explained by an increase in spontaneous activity (34). A further aim of the study was to investigate the lactate effect at the cellular level. Stimulated by the work of Watson et al. (44), we tried to distinguish between the two parameters, low $\mathrm{pH}$ and the lactate anion, in isolated hepatocytes of the $B$. marinus toad. We chose hepatocytes since the liver, as an aerobic organ with a high oxygen demand, comprises a significant fraction of standard metabolism of the whole animal [17\% in humans (39)]. Liver of another toad, Bufo americanus, was shown to accumulate a significant fraction of infused $\left[{ }^{14} \mathrm{C}\right]$ lactate and convert a high proportion into glycogen, protein, and lipid (46). Compared with the work by Watson et al. (44) on cardiomyocytes, our study should also reveal whether lactate might exert a stimulating effect on the metabolism of more than just one tissue or cell type. As a result, we report cellular mechanisms of an increase in metabolic rate induced by lactate that have so far not been considered in hypoxia-induced increments in metabolic rate and also in analyses of EPOC.

\section{MATERIALS AND METHODS}

Animals. B. marinus of either sex weighing between 220 and $350 \mathrm{~g}$ were obtained from a commercial dealer (P. Krauss, Mareeba, QLD, Australia). The toads were kept in large aquaria with a 15-cm layer of pet bedding and free access to water. They were force fed twice per week with chopped beef but were fasted 4 days before experimentation. The ethics committee of the Province of Bremen approved all experiments.

Respirometry. Toads were anaesthetized in tap water containing $2 \mathrm{~g} / \mathrm{l}$ 3-aminobenzoicacidethylester (MS-222, Sigma Chemical, St. Louis, MO; $\mathrm{pH}$ neutralized with $\mathrm{NaHCO}_{3}$ ). The ischiadic artery of the right hindleg was cannulated occlusively according to McDonald et al. (26). The animals were allowed to recover for $24 \mathrm{~h}$ in a darkened aquarium, and the experiment was started with a 12 -h period of acclimation to the respirometer chamber (2-liter volume, containing $200 \mathrm{ml}$ of water).
The inspired gas mixture was prepared from pure nitrogen and air with gas-mixing pumps (type $5 \mathrm{KM}$ 402/A-F, Wösthoff, Bochum, Germany). The oxygen content of the inspired gas mixture was reduced to $12 \%$ for $12 \mathrm{~h}$ and then to $7 \%$ for another $12 \mathrm{~h}$. This level of hypoxia elicited continuous breathing and thereby ensured a clear and immediate response to lactate infusions. It nonetheless provided sufficient oxygen for a fully aerobic metabolism $(34,36)$. Thirty-six hours after the start of the experiment, the animals were injected with isosmotic solutions of sodium lactate or $\mathrm{NaCl}$ (79 mmol/l, $24 \mathrm{mmol} / / \mathrm{NaHCO}_{3}, \mathrm{pH} 7.8,10 \mathrm{ml} / \mathrm{kg}$ body wt). All infusions were performed by using a microinjection pump (CMA 100, CMA Microdialysis, Stockholm, Sweden) at low speed $(100 \mu \mathrm{l} / \mathrm{min})$. As an example, a toad of $220 \mathrm{~g}$ was injected with $2.2 \mathrm{ml}$ over a period of $22 \mathrm{~min}$. Faster pump rates stimulated an increase in oxygen consumption. Low pump rates also minimized the risk of catecholamine release as a result of stress elicited by the injections. After $0.5,1,2$, 3,5 , and $7 \mathrm{~h}$, blood samples $(200 \mu \mathrm{l})$ were withdrawn via the indwelling catheter for lactate and catecholamine analyses in the blood plasma. The blood was centrifuged, and the supernatant plasma was removed. A stabilizing solution was added to one fraction of the plasma for catecholamine analyses $(20 \mu \mathrm{l} / \mathrm{ml}$ plasma, $25 \mu \mathrm{mol} / \mathrm{l} \mathrm{EGTA}, 0.5 \mathrm{mmol} / \mathrm{l}$ glutathione dissolved in $0.5 \mathrm{mmol} / \mathrm{l} \mathrm{NaOH}, \mathrm{pH} 7.0-7.5)$. The remaining plasma fraction was used for lactate analysis.

Oxygen consumption was measured with a paramagnetic oxygen and a photometric $\mathrm{CO}_{2}$ sensor (Oxynos-100 and $\mathrm{Bi}-$ nos-100, Rosemount). Data were recorded on a MacLab System (AD Instruments) and evaluated by using equations provided by Withers (45). Fast changes in oxygen consumption were calculated by repeatedly correcting for washout time over short intervals by using the equations of Bartholomew et al. (2), which take into account effective chamber volume and the rate of gas flow and calculate the equilibrium oxygen level of effluent gas that would be reached with no further changes in oxygen consumption (34). Oxygen consumption was recorded under hypoxia (7\% oxygen) before and for $7 \mathrm{~h}$ after injection. At the end of the experiments, the animals were anaesthetized and decapitated.

Analysis of catecholamines and lactate. Extraction of the catecholamines from the plasma was conducted by using a kit from Chromsystems for chromatographic separation with a HPLC system (Pharmacia) and electrochemical detection (EDC 41000, Chromsystems). The loss of epinephrine and norepinephrine due to the extraction procedure was determined by calibration standards (Chromsystems) and amounted to $11 \%$ on average. Detection limits for both epinephrine and norepinephrine were $10 \mathrm{pg} / \mathrm{ml}$.

Lactate levels in the plasma were measured enzymatically according to Bergmeyer (5) after extraction of the plasma or of the tissue of interest with perchloric acid, according to Beis and Newsholme (4).

Preparation of hepatocytes. Liver was quickly removed from freshly killed animals and washed in Ringer solution containing $1 \mathrm{mg}$ of BSA $/ \mathrm{ml}$ (composition in mmol/l: $79 \mathrm{NaCl}$, nominally $24 \mathrm{NaHCO}_{3}, 3.22 \mathrm{KCl}, 3.18 \mathrm{Na}_{2} \mathrm{HPO}_{4}, 1 \mathrm{CaCl}_{2}, 1$ $\mathrm{MgSO}_{4}, 5.55$ glucose, 10 HEPES, $\mathrm{pH}$ 7.4). Blood was removed by use of a Pasteur pipette inserted into the large vein and rinsing with Ringer solution. The tunica fibrosa was gently pulled off, and the tissue was transferred into $2.5 \mathrm{ml}$ of Ringer solution with BSA, where it was minced with two scissors. Crude collagenase (1,000 U/ml, Sigma Chemical) and hyaluronidase $(750 \mathrm{U} / \mathrm{ml}$, Merck) were added, and the digestion was supported by mechanical dispersion through repeated pipetting [preparation modified after Freshney (15)]. The perfusion method after Guguen-Guillouzo and 
Guillouzo (18) was not used because the enzymatic digestion technique yielded enough viable cells. Hepatocytes were separated from other cell types by centrifugation in a density gradient (20,000 revolutions/min, $30 \mathrm{~min}$, Rotor 42.1, Beckman L7-80 ultracentrifuge) using 45\% Percoll (Pharmacia) in Ringer solution. Hepatocytes were found between 1.060 and $1.075 \mathrm{~g} / \mathrm{ml}$. Hepatocytes were washed three times to remove all Percoll and counted on a hemocytometer (FuchsRosenthal) under a microscope (Leica DM LB, Bensheim) at 60 -fold magnification. Hepatocytes were identified according to size, since they are the largest cells in the liver. Viability was determined by their ability to exclude trypan blue.

The cells were kept in normoxic Ringer solution and allowed to recover for at least $5 \mathrm{~h}$ before experiments started. All cell suspensions were used within 2 days and were checked for viability before experimentation. Because of the short duration of the experiments, the use of antibiotics could be avoided. Bacterial contamination of the buffers was kept low by repeated sterile filtration and steam sterilization.

Measurements of cellular oxygen consumption. The oxygen consumption of the hepatocytes in several test solutions was measured by using fiber optic oxygen sensors (optodes, MOPS-4, Comte, Germany) starting from normoxic oxygen levels [i.e., hyperoxic compared with in vivo blood oxygen tensions under normoxia (36)]. The optodes allowed measurements in small volumes $(200 \mu \mathrm{l})$ such that repeated analyses could be carried out by using aliquots from one hepatocyte preparation.

The cells were spun down at low centrifugation speed (centrifuge 5402, Eppendorf) and resuspended in Ringer solution containing $1 \mathrm{mg} / \mathrm{ml}$ BSA with and without HEPES. Nonbicarbonate buffer values of Ringer solutions were $\sim 6.3$ $\mathrm{mmol} \cdot \mathrm{pH}^{-1} \cdot \mathrm{l}^{-1}$ with and $1.9 \mathrm{mmol} \cdot \mathrm{pH}^{-1} \cdot \mathrm{l}^{-1}$ without HEPES (at pH 7.0) compared with buffer values of plasma (4.1 $\left.\mathrm{mmol} \cdot \mathrm{pH}^{-1} \cdot \mathrm{l}^{-1}\right)$ and interstitial fluid $(\sim 0.4 \mathrm{mmol}$. $\left.\mathrm{pH}^{-1} \cdot \mathrm{l}^{-1}\right)(30,33,37)$. Solutions were set to variable lactate concentrations and to variable $\mathrm{pH}$ by using fixed acid or base for titration of Ringer solutions without cells. Solutions with relatively low buffer values as expected in interstitial fluid should support the development of $\mathrm{pH}$ gradients close to the cellular surface $(33,37)$ and thereby mimic the influence of interstitial fluid on cellular surface $\mathrm{pH}$. Before application, the inhibitors of $\mathrm{Lac}^{-}-\mathrm{H}^{+}$cotransport [ $\alpha$-cyano-4-hydroxycinnamate (CHC), $5 \mathrm{mmol} / \mathrm{l}$; Sigma Chemical] and $\mathrm{Na}^{+}-\mathrm{H}^{+}$ transport (EIPA, $100 \mu \mathrm{mol} / \mathrm{l}$, Sigma Chemical) were dissolved in Ringer solution at the appropriate $\mathrm{pH}$. After addition of the cell suspension, vials were sealed gas tight with dental periphery wax, which also kept the optode in place. Constant temperature $\left(20 \pm 0.5^{\circ} \mathrm{C}\right)$ was achieved by a temperature-controlled water bath and stirring of the cell suspension. Oxygen consumption was normalized to the protein content in each vial. Protein concentration was determined according to Bradford (7).

Statistics. Cellular oxygen consumption rates determined in samples from the same preparation of hepatocytes under control conditions and with various levels of lactate were tested for significant differences by using the paired-sample $t$-test and linear regression analyses. ANOVA (SuperANOVA, Abacus Concepts, 1991) was used for the comparison of more than one time point and of unpaired samples. Data were assumed to be significantly different at a significance level of $95 \%$.

\section{RESULTS}

Oxygen consumption of toads after isosmotic infusions. The original observation of a lactate effect on metabolic rate was under progressive hypoxia, when lactate formation set in below the $\mathrm{P}_{\mathrm{c}}(36)$. For a clear distinction between lactate and other hypoxia effects, toads in this and our laboratory's earlier study (34) were maintained under fully aerobic conditions at mildly hypoxic conditions. This forced the toads to breathe continuously, such that the response to lactate was not delayed or hidden due to intermittent breathing and apnea periods. Figure $1 A$ shows the changes in oxygen consumption in response to injected solutions. The oxygen consumption of aerobic $B$. marinus continuously breathing under mild hypoxia at rest was $2.16 \pm 0.41 \mathrm{mmol} \cdot \mathrm{h}^{-1} \cdot \mathrm{kg}^{-1}$. Sodium lactate caused a sudden $40 \%$ increase in oxygen consumption. After the infusion of $\mathrm{NaCl}$, oxygen consumption rose as well, but more slowly than after sodium lactate injections. During the first $60 \mathrm{~min}$, oxygen consumption under the effect of lactate was significantly higher than after the control injection of $\mathrm{NaCl}(P<0.05$, ANOVA). Accordingly, the time course of responses to lactate and $\mathrm{NaCl}$ started with a significant difference, which then turned into a nonsignificant difference. The two curves met after $4 \mathrm{~h}$, which reflected the continuous disappearance
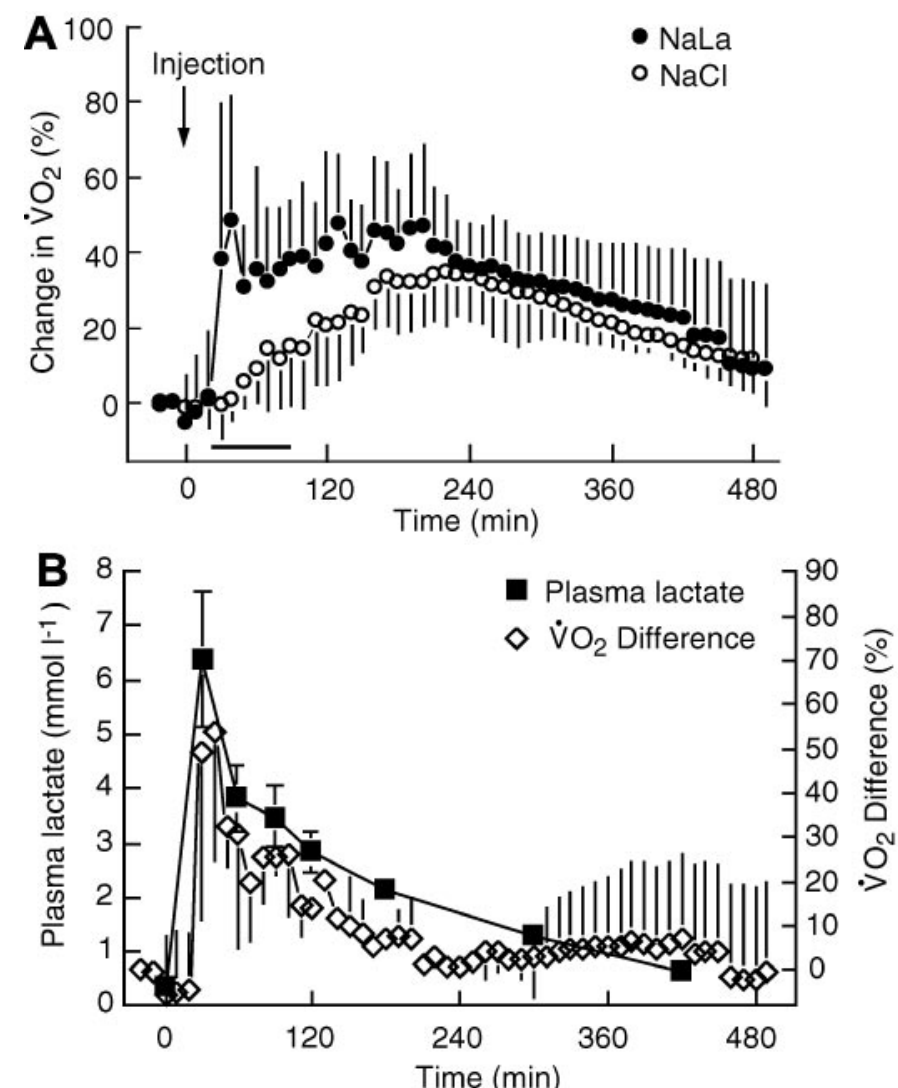

Fig. 1. A: oxygen consumption $\left(\dot{\mathrm{V}}_{2}\right)$ of Bufo marinus after injections of isosmotic sodium lactate $(\mathrm{NaLa})$ and $\mathrm{NaCl}$ solutions. The horizontal bar indicates a significant difference between the rise in oxygen consumption after injection of sodium lactate and the change seen after the control injection of $\mathrm{NaCl}$ (means $\pm \mathrm{SD}, n=6, P<0.05$, ANOVA). $B$ : plasma lactate concentrations after injection of isosmotic sodium lactate solutions ( $\mathbf{\square}$, means $\pm \mathrm{SD}, n=6$ ) compared with the drop in the difference between oxygen consumption rates after $\mathrm{NaCl}$ and sodium lactate injections $(\diamond)$. This difference approached zero when the lactate concentration fell below $1.5-2 \mathrm{mmol} / \mathrm{l}$. 
of the difference between the two patterns. Both curves decreased together toward control levels.

The time course of changes in plasma lactate levels is shown in Fig. $1 B$. Preinjection levels reflected fully aerobic conditions (36). Lactate reached a maximum concentration directly after the injection and decreased to control levels within $7 \mathrm{~h}$. This drop was paralleled by a decrease in the difference between oxygen consumption values found after sodium lactate and $\mathrm{NaCl}$ injections. This difference makes it possible to exclude effects that may have been elicited by the large injection volume. The figure shows a clear correlation between decreasing plasma lactate levels and the falling rate of oxygen consumption. The difference of the two oxygen consumption curves approached zero when plasma lactate fell below a concentration of $1.5-2 \mathrm{mmol} / \mathrm{l}$.

Catecholamine levels. No significant changes in epinephrine and norepinephrine concentrations were observed after the injection of isosmotic sodium lactate solutions. A transient trend for the concentration of epinephrine to increase was visible but remained insignificant $30 \mathrm{~min}$ after the injection of sodium lactate (Fig. 2). Mean levels had dropped to preinjection values within $1 \mathrm{~h}$, whereas lactate-induced oxygen consumption was still elevated over the consumption caused by $\mathrm{NaCl}$.

Cell viability. The number of hepatocytes obtained per animal ranged between 1 and $7.5 \times 10^{6}$. Of these cells, 96\% showed no staining with trypan blue and were, therefore, considered viable. Contamination with other cell types was by $<4 \%$. Cells remained viable for $>24 \mathrm{~h}$.

Lactate effects on cellular oxygen consumption. The nonrelease of catecholamines on lactate infusion and the work of Watson et al. (44) made us think that lactate may exert a direct effect on cellular metabolism through further, so far, unexplained mechanisms. Oxygen consumption of hepatocytes in HEPES-buffered Ringer solution was independent of the lactate concentration present and of extracellular $\mathrm{pH}$ in the range between 7.0 and 7.8 (Fig. 3A). No significant changes were observed. However, increasing lactate concentra-

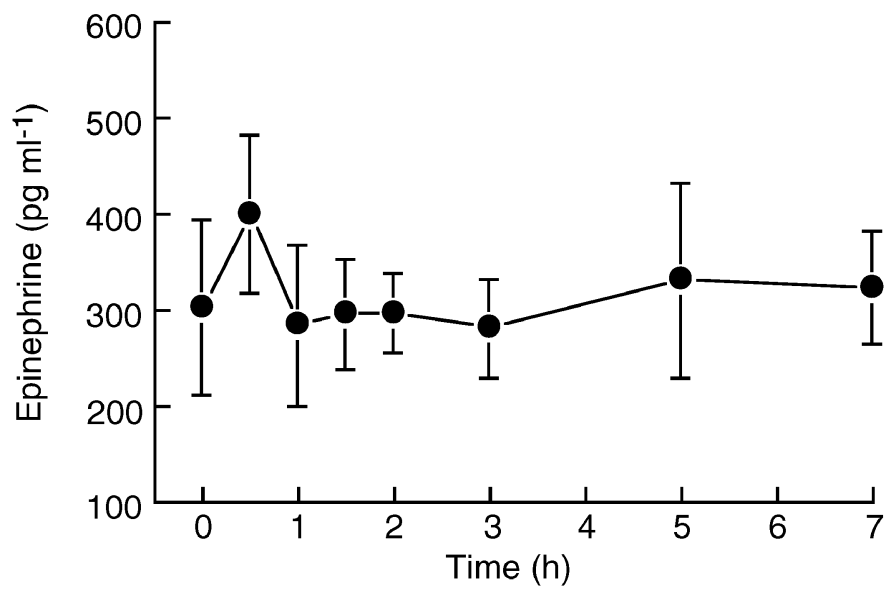

Fig. 2. Plasma epinephrine concentration after injection of isosmotic sodium lactate solutions. The transient increase seen after $30 \mathrm{~min}$ remained nonsignificant. Values are means $\pm \operatorname{SD}(n=6)$.
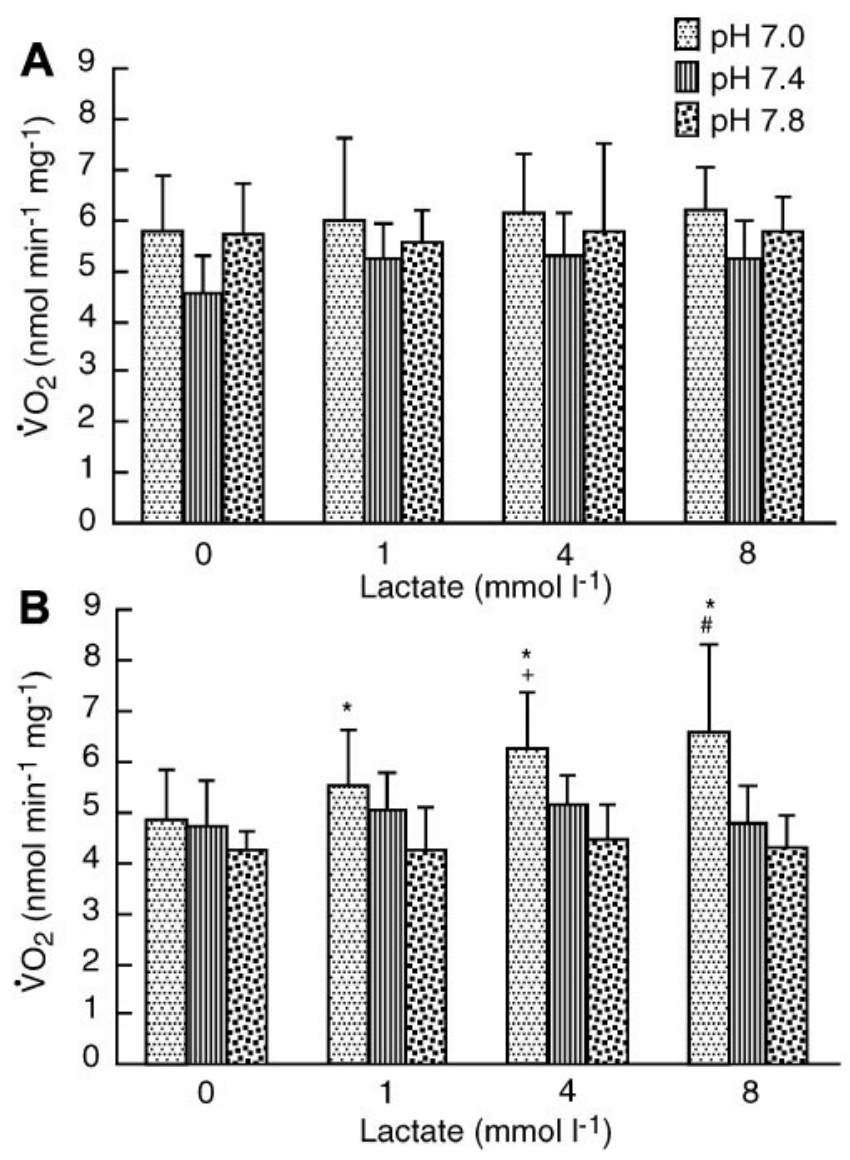

Fig. 3. A: $\dot{\mathrm{V}}_{2}$ of isolated hepatocytes in HEPES-buffered Ringer solution at different lactate concentrations and extracellular $\mathrm{pH}$ values (means $\pm \mathrm{SE}, n=6-13$ ). $B$ : oxygen consumption of isolated hepatocytes in weakly buffered Ringer solution at increasing lactate concentrations and various values of extracellular $\mathrm{pH}$. Values are means $\pm \mathrm{SE}(n=6-8)$. *Significantly different from control values without lactate; + ,\#significantly different from the effect of all lower lactate concentrations (paired sample $t$-test, $P<0.05$ ).

tions caused a significant rise in oxygen consumption in weakly buffered Ringer solution at low extracellular $\mathrm{pH}(\mathrm{pH}$ 7.0), whereas increasing lactate concentrations had no effect on oxygen consumption at $\mathrm{pH} 7.4$ and 7.8 (Fig. $3 B$ ). At $\mathrm{pH} 7.0$, a significant increase in oxygen consumption occurred at a lactate level of $1 \mathrm{mmol} / \mathrm{l}$. The metabolic increment rose parallel to the level of lactate at constant $\mathrm{pH}$. The highest lactate concentration applied $(8 \mathrm{mmol} / \mathrm{l})$ caused oxygen consumption to increase significantly by $35 \%$. The lactate-induced increase in oxygen consumption at $\mathrm{pH} 7.0$ could be completely prevented by inhibition of $\mathrm{Na}^{+} / \mathrm{H}^{+}$exchange in the presence of EIPA and at least partially by inhibition of $\mathrm{Lac}^{-}-\mathrm{H}^{+}$symport in the presence of $\alpha-\mathrm{CHC}$ (Fig. $4 A)$. Figure $4 B$ displays the significant increase in cellular respiration rates with rising lactate levels after an overproportional rise between 0 and $1 \mathrm{mmol}$ lactate/l.

\section{DISCUSSION}

Lactate-induced oxygen consumption in vivo. During hypoxia below the $\mathrm{P}_{c}$, oxygen consumption increases in 

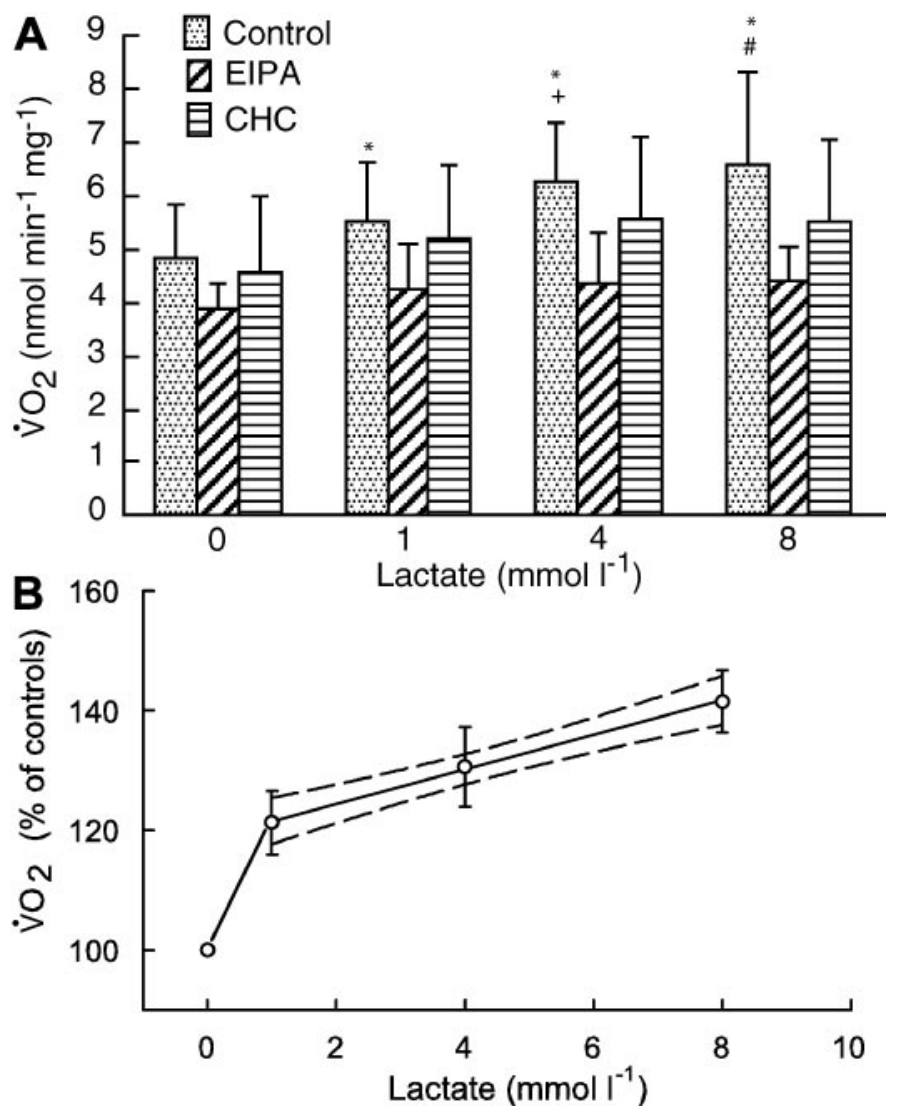

Fig. 4. $A: \dot{\mathrm{V}}_{2}$ of isolated hepatocytes in weakly buffered Ringer solution ( $\mathrm{pH} 7.0$ ) at rising lactate concentrations and under the effect of EIPA $(100 \mu \mathrm{mol} / \mathrm{l})$ and $\alpha$-cyano-4-hydroxycinnamate (CHC; 5 $\mathrm{mmol} / \mathrm{l})$. Both inhibitors prevented the increase in oxygen consumption caused by lactate. Values are means SE of $6-8$ subjects. * Significantly different from controls without lactate; +,\#significantly different from the effect of all lower lactate concentrations (paired sample $t$-test, $P<0.05$ ). $B$ : normalized levels of oxygen consumption of isolated hepatocytes (control conditions set to 100\%). After an initial overproportional rise in oxygen consumption, further addition of lactate resulted in a linear increase in metabolic rate. Dashed lines, $95 \%$ confidence interval. Values are means $\pm \mathrm{SE}\left(R^{2}=0.48\right.$; $P<0.05)$.

B. marinus despite progressive oxygen limitations. This increment occurs at the same time when lactate accumulation in the blood and tissues indicates the onset of net anaerobic metabolism in the whole animal. Insufficient oxygen supply to mitochondria is also indicated by succinate accumulation in heart ventricle (36). It must be emphasized here that the animal is not completely dependent on anaerobic metabolism when net lactate formation becomes detectable during progressive hypoxia, because not all tissues or cells of the animal (not all mitochondria) will become anaerobic at the same time. This leaves room for some aerobic scope. Systemic injections of an isosmotic sodium lactate solution in animals exposed to hypoxia just above the $\mathrm{P}_{\mathrm{c}}$ (Fig. 1) confirmed our previous findings with infusions of hyperosmotic solutions and demonstrated that lactate can elicit an increase in metabolic rate (Ref. 34 and the present study). After injection of comparatively high volumes of isosmotic sodium lactate solutions $[10 \mathrm{ml} / \mathrm{kg}$ vs. $5 \mathrm{ml} / \mathrm{kg}$ in previous experiments (34)], oxygen consumption rose to a similar maximum extent (by $40 \%$ ) to that after injection of hyperosmotic solutions [by $50 \%$ (33)], despite somewhat lower maximum plasma lactate levels (6.5 vs. $9 \mathrm{mmol} / \mathrm{l})$. Similar to previous observations (34), our present study also suggests that oxygen consumption remains elevated as long as the level of plasma lactate is above 1.5-2 $\mathrm{mmol} / \mathrm{l}$ (Fig. $1 B$ ) and decreases to control levels as soon as the concentration of lactate falls below this threshold value. In contrast to a rapid but short-term metabolic stimulation observed by Pörtner et al. (34) after infusion of a hyperosmotic $\mathrm{NaCl}$ solution, animals injected with a larger volume of isosmotic $\mathrm{NaCl}$ solution displayed a slow increase in oxygen consumption with a maximum reached after $4 \mathrm{~h}$. Here, the curve met the one recorded with sodium lactate, and both curves decreased together to preinjection levels. The strong reaction of the animals to isosmotic saline cannot be attributed to one of the ions, sodium or chloride, because then the animals should have displayed even stronger reactions to the hyperosmotic injections. Rather, the large injection volume required to obtain a sufficiently high plasma-lactate concentration by infusion of an isosmotic solution (see above) may have caused such a strong reaction despite the finely controlled slow infusion velocity. This interpretation is supported by the parallel development of both curves (saline vs. lactate infusions) over time after the lactate effect had vanished (Fig. 1).

In general, the metabolic breakdown of accumulated lactate cannot explain the increased metabolic rate under hypoxic conditions. Even after the injection of hyperosmotic lactate solutions (34), the increment was higher than required to convert all lactate present into glycogen.

In the present study, we investigated whether increased concentrations of plasma lactate cause or contribute to the release of catecholamines observed under severely hypoxic conditions $(1,23,27)$. Such a release might contribute to metabolic stimulation and would be in line with earlier results obtained by application of adrenergic blockers that prevented some but not all of the lactate-induced metabolic rate increment (34). A similar result was also found by Nedrow et al. (27) in desert iguanas after exercise. In these animals, $\alpha$ - and $\beta$-adrenergic blockade inhibited 25-40\% of the EPOC. It remains unclear to what extent lactate was involved in stimulating EPOC in the iguanas. In our study, metabolic stimulation of the resting animal occurred without a significant release of catecholamines after the injection of sodium lactate (Fig. 2). Norepinephrine, which was found to accumulate in the study by Andersen et al. (1), was not found in any of the samples. The nonsignificant trend for epinephrine to increase after the injection of isosmotic sodium lactate solutions might be related to a stress reaction of the animals, which proved to be very sensitive to injections at increased injection velocity (data not shown). As a corollary, the large accumulation of catecholamines observed under severely hypoxic conditions in $B$. $m a$ - 
rinus (1) may not or not exclusively depend on the rise in plasma lactate levels but may require additional stimuli associated with oxygen deficiency below the $\mathrm{P}_{\mathrm{c}}$ (present study, Ref. 35), which we excluded in our experiments for a clearer picture of the lactate effect. Nonetheless, local release or local effects of catecholamines, e.g., a stimulating effect of epinephrine via $\alpha$-adrenoceptors on $\mathrm{Na}^{+} / \mathrm{H}^{+}$exchange, and thus oxygen consumption, cannot be excluded and might explain why adrenergic blockers are effective in reducing the lactate-induced metabolic increment (34) with only minor changes in blood catecholamine levels (this study). Clearly, further study of local release and effects are needed to clarify the contribution of catecholamines to the effects of lactate in vivo.

Lactate-induced oxygen consumption in hepatocytes. With a possibly small contribution of catecholamines to the lactate effect in vivo, it appeared useful for us to look for further mechanisms that might contribute. In fact, further study revealed that, in addition to a potential local effect of catecholamines, lactate can directly increase oxygen consumption at the cellular level. In this context, unexplained changes in oxygen consumption have been found in oxygen debt analyses $(6,11,40)$ but have never been related to a direct effect of lactate. During oxygen debt after exercise (EPOC), the consumed oxygen exceeds the energetic requirements for glycogen anabolism and repletion of highenergy phosphate pools and presumably also the costs of increased ventilation and circulation.

Watson et al. (44) measured the oxygen consumption of cardiac myocytes under lactacidosis. At low bulk extracellular $\mathrm{pH}$, the authors found elevated metabolic rates that they attributed to $\mathrm{pH}_{\mathrm{i}}$ regulation via the $\mathrm{Na}^{+} / \mathrm{H}^{+}$exchanger, since the metabolic increment could be blocked by the addition of amiloride. The specific effect is not clear, because they did not compare this effect of amiloride to its effect on control metabolic rate (without lactate). We decided to investigate whether the transport of lactate into the cells might be involved. Hepatocytes in HEPES-buffered Ringer solutions with lactate at $\mathrm{pH}$ values of $\geq 7$ did not display an increased metabolic rate at all (Fig. 3A). Clearly, the presence of the lactate anion alone was not sufficient. Because lactate and protons are transported together across the plasma membrane, we assumed that the buffering of the Ringer solution by HEPES may minimize the buildup of a low enough cellular surface $\mathrm{pH}$ due to transmembrane $\mathrm{PCO}_{2}$ gradients (35). This may impair lactate uptake via the $\mathrm{Lac}^{-}-\mathrm{H}^{+}$transporter. Therefore, we repeated the experiments in weakly buffered Ringer solutions at the same bulk pH (Fig. 3B) and thereby simulated exposure of the cells to interstitial fluid in vivo. In this case, the cells displayed a rise in oxygen consumption depending on the lactate concentration present, however, only at low bulk extracellular $\mathrm{pH}$ (7.0). The difference between the effects of buffered and unbuffered Ringer solutions at $\mathrm{pH} 7.0$ implies that the $\mathrm{pH}$ in proximity to the cellular surface fell sufficiently only in unbuffered solution and that a low cellular surface $\mathrm{pH}$, likely $<7.0$, is required for the mediation of the lactate effect on metabolic rate.

Further experiments suggest that the $\mathrm{Lac}^{-}-\mathrm{H}^{+}$ transporter plays an important role in metabolic stimulation by lactate. $\alpha$-Cyanocinnamates are competitive inhibitors for these monocarboxylate transporters (29). These inhibitors were known already in 1974 (19), although the transporter was sequenced only recently (16), and a new family of transporter proteins is going to be defined (38). Inhibition by $\alpha$-cyanocinnamates usually remains incomplete. The largest degree of inhibition of lactate transport by $\alpha-\mathrm{CHC}$ in lower vertebrates (by 40\%) was described by Wang et al. (43) in skeletal muscle of rainbow trout, Oncorhynchus mykiss, and very recently in lizards by Donovan and Gleeson (13) with $42-54 \%$ inhibition of lactate uptake in red muscle tissue. Incomplete inhibition might reflect the competitive nature of the effect as well as nonionic diffusion of lactic acid across cellular membranes $(30,29)$. Although the affinity of the $\mathrm{Lac}^{-}-\mathrm{H}^{+}$ transporter in our hepatocytes for $\alpha-\mathrm{CHC}$ is not known, these observations may explain why the effect of $\alpha$-CHC remained below the effect of EIPA (Fig. 4).

Overall, our observations confirmed that the $\mathrm{Lac}^{-}-\mathrm{H}^{+}$transporter participates in the rise in oxygen consumption. In conclusion, both lactate and extracellular protons must be present in adequate concentrations for the mediation of an increase in metabolic rate. The difference between strongly vs. weakly buffered solutions may also lead to an explanation of why the infusion of sodium lactate stimulates metabolism in vivo despite slightly elevated values of plasma $\mathrm{pH}$ (34). $\mathrm{pH}$ values are likely lower in the interstitial fluid and even more so at the cellular surface than in the blood $(33,37)$, and this is most likely a prerequisite for the lactate effect to develop. A cellular surface $\mathrm{pH}$ of 7.0 or below may appear low; however, the very low buffer capacity of the interstitium allows for a considerable $\mathrm{pH}$ gradient over very short distances between the cellular surface and the plasma and even within the interstitium. For example, a $\mathrm{pH}$ of 0.2 units lower in interstitial fluid than in plasma already arises from the difference between bulk cellular and interstitial fluid $\mathrm{PCO}_{2}$ at rest (for further discussion, see Refs. 30, 37). Even lower values are conceivable at the cellular surface, especially during metabolic stimulation with elevated levels of $\mathrm{CO}_{2}$ production. Exercise caused arterial blood $\mathrm{pH}$ to fall from 7.8 to 7.4 and $\mathrm{PCO}_{2}$ to rise from $\sim 12$ to 17 Torr at close to $8 \mathrm{mM}$ plasma lactate in B. marinus (26). A 0.3 - to 1 -unit difference in $\mathrm{pH}$ between plasma and interstitial fluid would cause 2 to 10 times higher undissociated lactic acid levels in the interstitial fluid than in the plasma and thereby stimulate lactate proton symport. Earlier observations in rat hepatocytes have, in fact, demonstrated a stimulation of net lactate proton uptake by low extracellular $\mathrm{pH}$ (14). Moreover, at a cellular surface $\mathrm{pH}$ below reported levels of $\mathrm{pH}_{\mathrm{i}}(37)$, higher intra- than extracellular lactate anion levels result according to weak acid distribution characteristics (30). The enhanced dissociation of lactic acid inside the cell supports an inward 
diffusion gradient of undissociated lactic acid, which further enhances the rate of lactic acid uptake and finally sodium proton exchange (Fig. 5). Because of the methodological problem of measuring $\mathrm{pH}$ and $\mathrm{PCO}_{2}$ gradients in weakly buffered solutions and in interstitial space, true surface $\mathrm{pH}$ values of the hepatocytes are not known. We cannot exclude the possibility that hepatocytes are special with respect to the level of $\mathrm{pH}$ required for the stimulation of lactate proton symport or the threshold level of the lactate effect on respiration. Accordingly, the same relationships need investigation in cellular preparations from other tissues. However, comparison with the work on cardiomyocytes by Watson et al. (44) and the similarity of the percent metabolic increment seen in our cellular preparations and in vivo suggests that these principles are operative in tissues other than liver as well.

Considering nonspecific side effects of $\alpha$-cyanocinnamates, $\alpha$-CHC might be transported into the intracellular space, causing an inhibition of the mitochondrial pyruvate carrier (19) and, in consequence, a drop in oxygen consumption. However, such an effect must be considered unlikely since we also found that, in hepatocytes, alanine induces metabolic stimulation

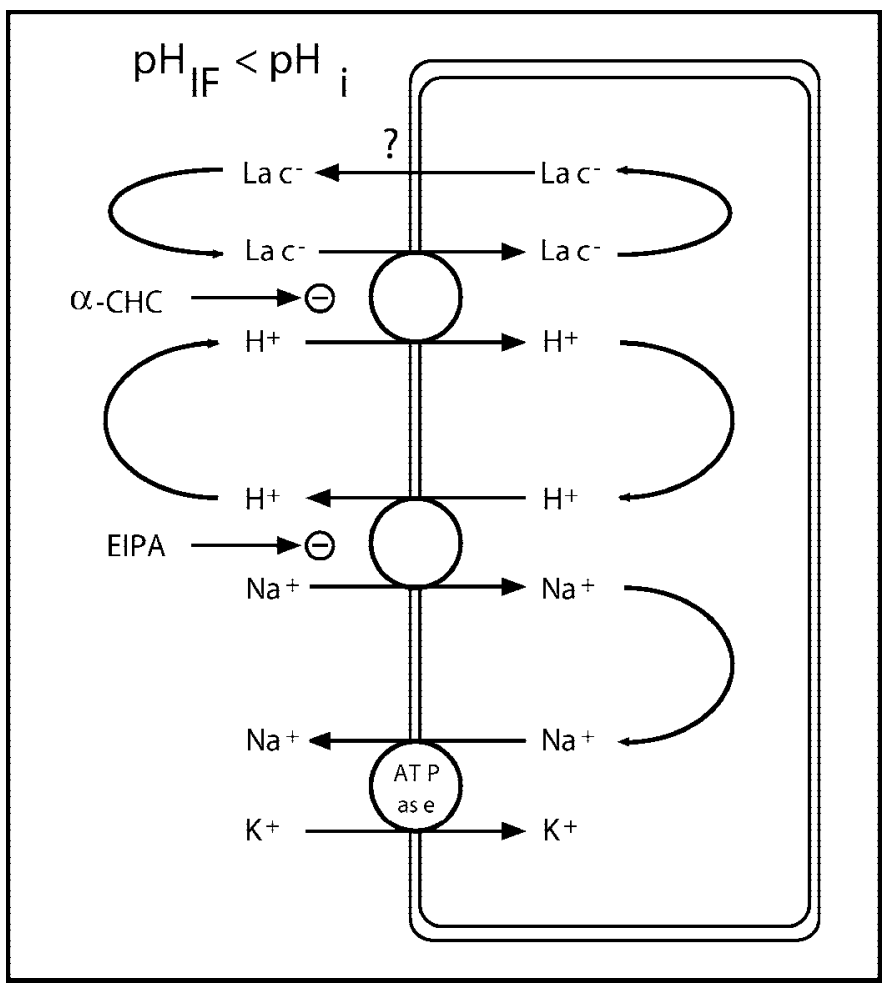

Fig. 5. Model illustrating the mechanism of the lactate-induced rise in cellular oxygen consumption. Lactate and proton symport across the plasma membrane activate the $\mathrm{Na}^{+}-\mathrm{H}^{+}$transporter and, in consequence, the $\mathrm{Na}^{+}-\mathrm{K}^{+}$-ATPase, thereby causing an increase in oxygen consumption. The latter can be prevented by inhibition of the lactate proton transporter $(\alpha-\mathrm{CHC})$ as well as by inhibition of $\mathrm{Na}^{+} / \mathrm{H}^{+}$exchange (EIPA). When cellular surface $\mathrm{pH}$ [interstitial $\mathrm{pH}$ $\left(\mathrm{pH}_{\mathrm{IF}}\right)$ ] falls below intracellular $\mathrm{pH}\left(\mathrm{pH}_{\mathrm{i}}\right)$, the intracellular accumulation of lactate anions and their release (e.g., by anion exchange) reflects "uncoupling" of the membrane, which is suggested to stimulate oxygen consumption even further (see text) that remains unaffected by $\alpha-\mathrm{CHC}$, although part of the alanine is likely deaminated to pyruvate (J. Pinz and H. O. Pörtner, unpublished results). We conclude that, even if the mitochondrial carrier was inhibited by $\alpha-\mathrm{CHC}$, this effect did not lead to a change in oxygen consumption.

The model displayed in Fig. 5 illustrates the lactateinduced increase in oxygen consumption. The symport of lactate and protons is likely to be responsible for extracellular alkalization (owing to proton removal) and concomitant intracellular acidification (present study, Ref. 34) that stimulates the $\mathrm{Na}^{+} / \mathrm{H}^{+}$exchanger (44) and, in consequence, $\mathrm{Na}^{+}-\mathrm{K}^{+}$-ATPase, reflecting a rise in energy turnover. Close coupling of $\mathrm{Na}^{+} / \mathrm{H}^{+}$ exchanger and $\mathrm{Na}^{+}-\mathrm{K}^{+}$-ATPase with respect to changes in oxygen consumption has recently been demonstrated in invertebrate muscle tissue (Ref. 32, during acidosis) and toad (B. marinus) hepatocytes (under alanine, H.-O. Pörtner and J. Pinz, unpublished data) and has also formed the basis of conclusions in various other studies $(21,44)$. EIPA as an amiloride derivative specifically inhibits $\mathrm{Na}^{+} / \mathrm{H}^{+}$exchange $(22,24)$ and prevents the increase in oxygen consumption (Fig. 4).

With the use of calculations made by Pörtner et al. (34), a maximum yield of $33 \mathrm{mmol}$ ATP/ $\mathrm{kg}$ is provided by the extra oxygen consumed in vivo after infusion of $4 \mathrm{mmol}$ lactate/kg. [The calculation assumed a P/O, i.e., phosphate-to-oxygen ratio, of 2.5, which includes some contribution of mitochondrial proton leakage to standard metabolism (10).] With an ATP requirement of $0.33 \mathrm{ATP} / \mathrm{H}^{+}$ion, irreversible lactate transport into the cell and subsequent removal of protons by sodium proton exchange (Fig. 4) would only consume a very small fraction $(1.3 \mathrm{mmol}$ ATP $/ \mathrm{kg})$ of the extra ATP provided. The effect of catecholamines in the systemic response does not make up for this discrepancy last but not least because it enhances the velocity of lactate removal (34). The contributions of lactate transport and sodium proton exchange to cellular metabolic stimulation according to Fig. 4 might therefore be much larger in vivo than expected from the stoichiometric and irreversible uptake of lactate. Together with the finding of a threshold value for the systemic response, which is in line with the overproportional rise in cellular respiration at low lactate levels (Fig. 4B), our observations exclude a direct stoichiometric relationship between the increase in oxygen consumption and the net amount of lactate cleared from the extracellular space. Accordingly, costs of lactate-induced acidbase regulation were probably nonstoichiometric in our cellular preparation too, indicated first by the overproportional rise in cellular oxygen consumption at low lactate levels (Fig. 4B) and second by persistent metabolic stimulation even at low lactate levels. The same levels of alanine only led to a shorter-term, transient stimulation of oxygen consumption that ended when all alanine was removed (H.-O. Pörtner and T. Hinse, unpublished observations).

The apparent discrepancy between expected and actual contributions of the transport mechanisms to the extra ATP demand suggests that the model developed 
so far remains incomplete with respect to the whole animal situation and possibly also for the hepatocytes. A testable hypothesis would be that the elevation of intracellular above extracellular levels of the lactate anion that results at low cellular surface $\mathrm{pH}$ might be compensated for by an as yet unidentified mechanism. Combined with net proton release via sodium proton exchange, this mechanism is equivalent to a partial "uncoupling" of the cellular membrane with respect to $\mathrm{pH}$ and, as long as elevated lactate levels and lower cellular surface than $\mathrm{pH}_{\mathrm{i}}$ values persist, would cause higher turnover rates of lactate proton symport and sodium proton exchange than expected from unidirectional movements of lactate and protons alone (Fig. 5).

In support of this hypothesis, evidence is accumulating that more than one mechanism is involved in lactate exchange between intra- and extracellular space. Some evidence for an anion exchange mechanism different from lactate proton symport exists for rat hepatocytes (39). A cycling of lactate similar to the one suggested in Fig. 5 has recently been observed in white muscle sarcolemmal vesicles prepared from rainbow trout (25). Unexpectedly, the level of lactate reached in the vesicular lumen over time was enhanced (not reduced) under 4-acetamido-4'-isothiocyanostilbene-2,2' disulfonic acid and even $\mathrm{CHC}$, suggesting carrier-mediated export of lactate from muscle. In $B$. marinus hepatocytes, an anion antiport (e.g., lactate/chloride exchange) would favor an outward movement of lactate anions, thereby causing a deviation from weak acid distribution in similar ways to those observed in rat hepatocytes $(27,37,39)$. Such an anion exchange mechanism may also be the basis for the frequently suggested trend toward a membrane potential-dependent distribution of lactate anions. Evidently, the functional integration of the various monocarboxylate transporters present in this and other cell types is still incompletely understood. Therefore, further study is required for a complete identification of the mechanisms involved in lactate movements in hepatocytes as well as in other organs involved in lactate metabolism (46).

It is conceivable that lactate-induced metabolic stimulation is not only operative when lactate is formed during progressive hypoxia below the critical oxygen tension (36) but also when lactate accumulates during exercise. Improved oxygen supply during recovery from exercise and the depression of plasma, interstitial, and cellular surface $\mathrm{pH}$ might then support full expression of the response in vivo. The increase in oxygen consumption after exercise (oxygen debt or EPOC) is still not fully understood, since the use of lactate in gluconeogenesis and the repletion of high-energy phosphates are again unable to account for up to $70 \%$ of the elevated metabolic rate in ecto- and endotherms (Refs. $6,27,40$, and references therein). The energetic costs of lactate-induced acid-base regulation may fill some of the unexplained gap in oxygen debt.

The data reported by Gleeson and Dalessio (17) support this interpretation. They investigated the fate of lactate after exercise in the lizard Dipsosaurus dorsalis and found an increased oxygen consumption rate that did not return to control levels in the investigated time period. At the same time, the lactate concentration did not return to control levels but remained significantly elevated. Later on, the same group demonstrated that catecholamines significantly contribute to EPOC (27), which is reduced before complete removal of lactate. The contribution of catecholamines as well as the existence of an extracellular threshold level for the lactate effect (34) and its dependence on the level of cellular surface $\mathrm{pH}$ would explain why the correlation between lactate levels and the degree of EPOC (40) or hypoxia-induced metabolic stimulation $(34,36)$ is poor.

Conclusions and perspectives. The rise in cellular metabolic rate induced by lactate shows a significant contribution of sodium proton exchange. Stimulation of this exchanger occurs by stimulation of $\mathrm{Lac}^{-}-\mathrm{H}^{+}$symport. The model (Fig. 5) suggests that metabolic stimulation by lactate and, accordingly, the contribution of this phenomenon to EPOC can vary depending on the established gradients between intracellular and cellular surface $\mathrm{pH}$, and it will also vary depending on the level of expression of the monocarboxylate transporters. Overall, the presented data substantiate cellular mechanisms likely involved in metabolic stimulation at the whole animal level. However, both the cellular and the whole animal picture still need to be complemented because the relationships between $\mathrm{pH}$ and metabolic stimulation may differ between cell and tissue types and because local release and effects of catecholamines need to be considered. For the whole animal, it also remains unclear how the lactate effect at the cellular level relates to the onset of behavioral hypothermia, which is elicited by lactate and occurs in parallel to the increase in metabolic rate $(8,34)$. In general and as a corollary, it appears very conceivable that a rise in the cost of $\mathrm{pH}_{\mathrm{i}}$ regulation represents one of the so far unexplained components in lactate-induced metabolic stimulation during hypoxia as well as during EPOC. The molecular and functional picture of the mechanisms involved still needs to be complemented and the relative and quantitative contributions of all of these mechanisms needs to be elaborated in further studies of hypoxia (below $\mathrm{P}_{\mathrm{c}}$ ) and exercise effects at the whole animal level.

Present address of I. Pinz: Brigham and Women's Hospital, Harvard Medical School, Boston, MA 02115.

\section{REFERENCES}

1. Andersen JB, Jensen FB, and Wang T. Effects of temperature and oxygen availability on circulating catecholamines in the toad Bufo marinus. Comp Biochem Physiol A 129: 473-486, 2001.

2. Bartholomew GA, Vleck D, and Vleck CM. Instantaneous measurement of oxygen consumption during pre-flight warm-up and post-cooling in sphingid and saturniid moths. J Exp Biol 90: 17-32, 1981.

3. Beamish FWH. Respiration of fishes with special emphasis on standard oxygen consumption. III. Influence of oxygen. Can $J$ Zool 42: 355-366, 1964.

4. Beis I and Newsholme EA. The contents of adenine nucleotides, phosphagens and some glycolytic intermediates in resting muscles from vertebrates and invertebrates. Biochem $J$ 152: 23-32, 1975 . 
5. Bergmeyer HU. Methods of Enzymatic Analysis (3rd ed.). Weinheim, Germany: Verlag Chemie, 1985, vols. 1-12.

6. Borsheim E, Knardahl S, Hostmark AT, and Bahr R. Adrenergic control of post-exercise metabolism. Acta Physiol Scand 162: 313-323, 1998.

7. Bradford MM. A rapid and sensitive method for the quantitation of microgram quantities of protein utilizing the principle of protein-dye binding. Anal Biochem 72: 248-254, 1976.

8. Branco LGS and Steiner AA. Central thermoregulatory effects of lactate in the toad Bufo paracnemis. Comp Biochem Physiol A 122: 457-461, 1999.

9. Branco LGS and Wood SC. Role of central chemoreceptors in behavioral thermoregulation of the toad, Bufo marinus. Am $J$ Physiol Regul Integr Comp Physiol 266: R1483-R1487, 1994.

10. Brand MD, Chien LF, Ainscow EK, Rolfe DFS, and Rolfe RK. The causes and functions of mitochondrial proton leak. Biochim Biophys Acta 1187: 132-139, 1994.

11. Brooks GA and Gaesser GA. End point of lactate and glucose metabolism after exhaustive exercise. J Appl Physiol 49: 10571069, 1980.

12. DeWachter B, Sartoris FJ, and Pörtner HO. The anaerobic endproduct lactate has a behavioural signaling function in the shore crab Carcinus maenas. J Exp Biol 200: 1015-1024, 1997.

13. Donovan ER and Gleeson TT. Evidence for facilitated lactate uptake in lizard skeletal muscle. J Exp Biol 204: 4099-4106, 2001.

14. Farfournoux P, Demigne C, and Remesy C. Carrier-mediated uptake of lactate in rat hepatocytes. Effects of $\mathrm{pH}$ and possible mechanisms for L-lactate transport. J Biol Chem 260: 292-299, 1985.

15. Freshney IR. Culture of Animal Cells. A Manual of Basic Technique (3rd ed.). New York: Wiley-Liss, 1994.

16. Garcia CK, Goldstein JL, Pathak RK, Anderson RGW, and Brown MS. Molecular characterization of a membrane transporter for lactate, pyruvate and other monocarboxylates: implication for the Cori cycle. Cell 76: 865-873, 1994.

17. Gleeson TT and Dalessio PM. Lactate and glycogen metabolism in the lizard Dipsosaurus dorsalis following exhaustive exercise. J Exp Biol 144: 377-393, 1989.

18. Guguen-Guillouzo C and Guillouzo A. Methods for preparation of adult and fetal hepatocytes. In: Isolated and Cultured Hepatocytes, edited by Guguen-Guillouzo $\mathrm{C}$ and Guillouzo A. Paris: Les Editions INSERM, 1986, p. 1-12.

19. Halestrap AP and Denton RM. Specific inhibition of pyruvate transport in rat liver mitochondria and human erythrocytes by

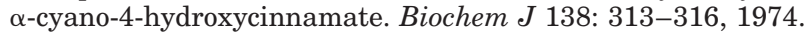

20. Halestrap AP and Denton RM. The specificity and metabolic implications of the inhibition of pyruvate transport in isolated mitochondria and intact preparations by $\alpha$-cyano-4-hydroxycinnamate and related compounds. Biochem $J$ 148: 97-106, 1975.

21. Harvitt DM and Bonanno JA. $\mathrm{pH}$ dependence of corneal oxygen consumption. Invest Ophthalmol Vis Sci 39: 2778-2781, 1998.

22. Johnson JD and Epel D. Intracellular pH and activation of sea urchin eggs after fertilisation. Nature 262: 661-664, 1976.

23. Kinkead R, Fritsche R, Perry SF, and Nilsson S. The role of circulating catecholamines in the ventilatory and hypertensive responses to hypoxia in the atlantic Cod (Gadus morhua). Physiol Zool 64: 1087-1109, 1991.

24. Kleyman TR and Cragoe EJ. Amiloride and its analogs as tools in the study of ion transport. J Membr Biol 105: 1-21, 1988.

25. Laberee $\mathbf{K}$ and Milligan CL. Lactate transport across sarcolemmal vesicles isolated from rainbow trout white muscle. $J$ Exp Biol 202: 2167-2175, 1999.

26. McDonald DG, Boutilier RG, and Toews DP. The effects of enforced activity on ventilation, circulation and blood acid-base balance in the semi-terrestrial anuran, Bufo marinus. J Exp Biol 84: 273-287, 1979.

27. Nedrow JM, Scholnick DA, and Gleeson TT. Roles of lactate and catecholamines in the energetics of brief locomotion in an ectothermic vertebrate. J Comp Physiol B Biol Sci 171: 237-245, 2001.

28. Perry SF and Gilmour KM. Consequences of catecholamine release on ventilation and blood oxygen transport during hypoxia and hypercapnia in an elasmobranch and a teleost. $J$ Exp Biol 199: 2105-2118, 1996.

29. Poole RC and Halestrap AP. Transport of lactate and other monocarboxylates across mammalian plasma membranes. Am J Physiol Cell Physiol 264: C761-C782, 1993.

30. Pörtner HO. Multicompartmental analyses of acid-base and metabolic homeostasis during anaerobiosis: invertebrate and lower vertebrate examples. In: Surviving Hypoxia: Mechanisms of Control and Adaptation, edited by Hochachka PW, Lutz PL, Sick T, Rosenthal M, and van den Thillart G. Boca Raton, FL: CRC, 1993, p. 139-156.

31. Pörtner HO. $\mathrm{pH}$ homeostasis in terrestrial vertebrates: a comparison of traditional and new concepts. Adv Comp Environ Physiol 22: 51-62, 1995.

32. Pörtner HO, Boutilier RG, Tang Y, and Toews DP. Determination of intracellular $\mathrm{pH}$ and $\mathrm{PCO}_{2}$ after metabolic inhibition by fluoride and nitrilotriacetic acid. Respir Physiol 81: 255-274, 1990.

33. Pörtner HO, Bock C, and Reipschläger A. Modulation of the cost of $\mathrm{pH}_{\mathrm{i}}$ regulation during metabolic depression: a ${ }^{31} \mathrm{P}-\mathrm{NMR}$ study in invertebrate (Sipunculus nudus) isolated muscle. J Exp Biol 203: 2417-2428, 2000.

34. Pörtner HO, Branco LGS, Malvin GM, and Wood SC. A new function for lactate in the toad, Bufo marinus. J Appl Physiol 76: 2405-2410, 1994.

35. Pörtner $\mathrm{HO}$ and Grieshaber MK. Critical $\mathrm{Po}_{2} \mathrm{~S}$ in oxyconforming and oxyregulating animals: gas exchange, metabolic rate and the mode of energy production. In: The Vertebrate Gas Transport Cascade: Adaptations to Environment and Mode of Life, edited by Bicudo JEPW. Boca Raton, FL: CRC, 1993, p. 330-357.

36. Pörtner HO, MacLatchy LM, and Toews DP. Metabolic responses of the toad Bufo marinus to environmental hypoxia: an analysis of the critical $\mathrm{PO}_{2}$. Physiol Zool 64: 836-849, 1991.

37. Pörtner HO, MacLatchy LM, and Toews DP. Acid-base regulation in the toad Bufo marinus during environmental hypoxia. Respir Physiol 85: 217-230, 1991.

38. Price NT, Jackson VN, and Halestrap AP. Cloning and sequencing of four new mammalian monocarboxylate transporter (MCT) homologues confirms the existence of a transporter family with an ancient past. Biochem $J$ 329: 321-328, 1998.

39. Rolfe DFS and Brown GC. Cellular energy utilization and molecular origin of standard metabolic rate in mammals. Physiol Rev 77: 731-758, 1997.

40. Scarabello M, Heigenhauser GJ, and Wood CM. The oxygen debt hypothesis in juvenile rainbow trout after exhaustive exercise. Respir Physiol 84: 245-259, 1991.

41. Talbot CR and Stiffler DF. Effects of hypoxia on acid-base balance, blood gases, catecholamines and cutaneous ion exchange in the larval tiger salamander (Ambystoma tigrinum). $J$ Exp Zool 257: 299-305, 1991.

42. Ultsch GR, Ott ME, and Heisler N. Standard metabolic rate, critical oxygen tension, and aerobic scope for spontaneous activity of trout (Salmo gairdneri) and carp (Cyprinus carpio) in acidified water. Comp Biochem Physiol A 67: 329-335, 1980.

43. Wang Y, Wright PM, Heigenhauser GJF, and Wood SM. Lactate transport by rainbow trout white muscle: kinetic characteristics and sensitivity to inhibitors. Am J Physiol Regul Integr Comp Physiol 272: R1577-R1587, 1997.

44. Watson CL, Walter LF III, Panol G, and Jackson DC. Lactic acidosis transiently increases metabolic rate of turtle myocytes. Am J Physiol Regul Integr Comp Physiol 266: R1238-R1243, 1994.

45. Withers PC. Measurement of $\dot{\mathrm{V}}_{2}, \dot{\mathrm{V}}_{\mathrm{CO}_{2}}$, and evaporative water loss with a flow-through mask. J Appl Physiol 42: 120-123, 1977.

46. Withers PC, Lea M, Solberg TC, Baustian M, and Hedrick M. Metabolic fates of lactate during recovery from activity in an anuran amphibian, Bufo americanus. J Exp Zool 246: 236-243, 1988. 